\title{
Gene knockout by inducing P-element transposition in Drosophila
}

\author{
H.L. Ou ${ }^{1,2}$ \\ ${ }^{2}$ Department of Biochemistry and Molecular Biology, \\ Guiyang Medical University, Guiyang, China \\ Corresponding author: H.L. Ou \\ E-mail: ouhailong@gmc.edu.cn \\ Genet. Mol. Res. 12 (3): 2852-2857 (2013) \\ Received May 9, 2012 \\ Accepted September 6, 2012 \\ Published January 4, 2013 \\ DOI http://dx.doi.org/10.4238/2013.January.4.20
}

${ }^{1}$ State Key Laboratory of Biomembrane and Membrane Biotechnology, Institute of Zoology, Chinese Academy of Sciences, Beijing, China

\begin{abstract}
The P-element has been successfully used in germline transformation to create transgenic flies and as an insertion mutagenesis agent to disrupt genes. Moreover, P-element can also be used to knockout genes that are near the insertion sites by inducing its imprecise transposition. In this article, P-element insertion lines were crossed with transposase expression fly to recover the transposon, and the deletion of flanking sequence, which was created by imprecise excision, was detected by PCR. Through this method, null alleles of seven genes that spread over three major chromosomes (X, second, third) were generated in Drosophila melanogaster. Results show that the frequency of flanking deletions is expected to be $1 \%$, and it is enough to pick up at least one ideal deletion line from 200 independent recovery lines in general. These data suggest that although the frequency of disrupted gene varied greatly, from 0.13 to $2.34 \%$, gene knockout by inducing P-element transposition appears to be a feasible and effective strategy, compared to the complicated process of gene targeting based on homologous recombination.
\end{abstract}

Key words: Gene knockout; P-element; Deletion; Transposition; Imprecise excision 


\section{INTRODUCTION}

The P-element was discovered as an autonomous transposable element in Drosophila; it has a complete sequence of $2907 \mathrm{bp}$ in length with a 31-bp terminal inverted repeat and an 11-bp subterminal inverted repeat (O'Hare and Rubin, 1983). P-elements encode a functional transposase that enables them to move by a cut-and-paste mechanism inside a genome, and are located between the two 11-bp subterminal inverted repeats. Owing to the pioneering study of Rubin and Spradling (1982), the P-element has become a common and important tool for germline transformation to generate transgenic flies. In our routine use, P-elements were integrated into plasmid vectors and submitted to further modifications to prevent uncontrolled transposition, including that the transposase gene is disrupted, where an interesting gene is inserted. Mediated by the P-element, the foreign gene is then integrated into the fly genome with high efficiency and stability. After crossing with a controlled transposase expressed line, the P-element could excise from the genome in their offspring. The excision can occur by a precise or imprecise method in most transposons including P-elements (O'Brochta et al., 1991). When a P-element is excised from the original insertion site, the chromosome is left with a double-strand break. If the ends of such break are degraded before repair, a deletion of the genetic material will be generated. Imprecise excision may have profound effects on the activity of adjacent genes, and genome stability.

P-element transposons have been successfully used as insertional mutagenesis to disrupt genes (Cooley et al., 1988). The Berkeley Drosophila Genome Project has collected about 7140 lines, which predicted the disruption of 5362 of the 13,666 currently annotated Drosophila genes (39\%) (Spradling et al., 1999). However, the insertion biases make it unlikely that full genome saturation would be reached using P-element mutagenesis despite the growing number of P-element collected in the Bloomington Stock Center. There are still sizable genes, where their function cannot be completely abolished, especially if the P-element is inserted in regions such as UTRs, introns, or non-gene regions. Imprecise excision can serve as a valuable tool to generate the null allele of such genes. Recently, we have produced a knockout of a series of genes that are distributed over all three major chromosomes (X, second, third) by inducing local P-element transposition. Here, the steps of the gene disruption approach that makes use of the imprecise excision mechanism of P-elements are described in detail.

\section{MATERIAL AND METHODS}

\section{Experimental design}

In the procedure, the $\mathrm{P}\{r y+\Delta 2-3\}(99 \mathrm{~B})$ element, abbreviated $\Delta 2-3$, was used as a source of transposase for mobilization of inserted P-elements near the target gene region. In the $\Delta 2-3$, the last intron of transposase was deleted in vitro, which makes it functional both in germline and somatic cells. While the element mediated the transposition of other nonautonomous P-elements with high frequencies, the element itself remains remarkably stable (Robertson et al., 1988). All insertion P-element lines used in this study contain the mini-white gene, thus they have pigmented eyes. The crossing scheme for recovering the P-element to delete the flanking sequences is described below (Figure 1):

Scheme 1. For P-elements located on the 1st chromosome, the P-element line was crossed with transposase source line $w / y ; \Delta 2-3, S b / T M 6 B, T b$ to generate $\mathrm{F}_{1}$ males $P / Y ; \Delta 2-3, S b /+$ 
which have mosaic red eye color. About $300-400$ crosses of single $F_{1}$ males carrying both the P-element and $\Delta 2-3$ chromosomes with $3 w^{+} / F m 7$ virgin females were carried out, followed by selection of the white eye $\triangle P / F M 7$ and $F M 7 / Y$ to establish excision mutation stocks.

A

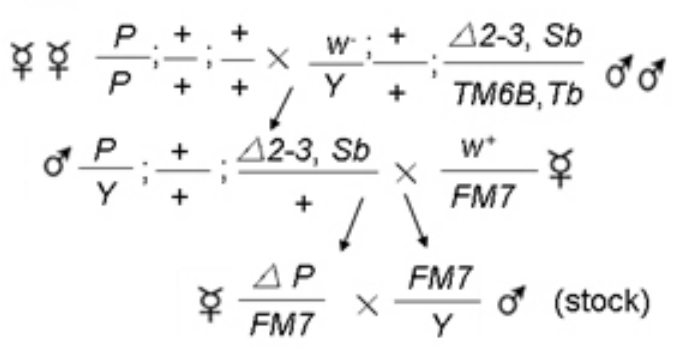

$\mathrm{C}$

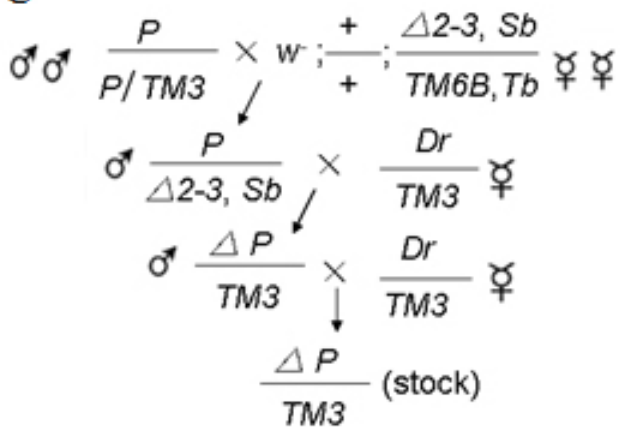

$\mathrm{B}$

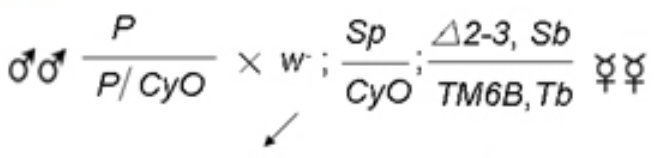
$\sigma^{*} \frac{P}{C y O / S p} ; \frac{\Delta 2-3, S b}{+} \times \frac{S c o}{C y O} \not{\gamma}$ $\sigma^{7} \frac{\Delta P}{C y O} \times \frac{S c o}{C^{\prime} y}$ 후 $\frac{\triangle P}{C y O}($ stock $)$

Figure 1. Genetic crosses for gene knockout through P-element imprecise excision. Target genes are located on first $(\mathbf{A})$, second (B), and third chromosomes $(\mathbf{C}) . P$ stands for inserted P-element; $\Delta P$ stands for the flanking sequences deleted after P-elements jumped out.

Scheme 2. For P-elements located on the 2nd chromosome, the P-element line was crossed with fly genotype $w ; S p / C y O ; \Delta 2-3, S b / T M 6 B, T b$ to obtain the $P / \Delta 2-3$ fly. After crossing the 300-400 individual $P / C y O ;+/ \Delta 2-3$ and $3 \mathrm{Sco} / \mathrm{Cy} O$ flies, 1 white eye male progeny $\triangle P /$ $\mathrm{CyO}$ per vial was picked up, and single-crossed with $3 \mathrm{Sco} / \mathrm{CyO}$ virgin females to establish about $200 \triangle P / C y O$ stocks.

Scheme 3. For P-elements located on the 3rd chromosome, the mosaic red eye fly $300-$ $400 \mathrm{P} / \Delta 2-3$ was created by mating the P-element line with transposase source line $w ; \Delta 2-3, \mathrm{Sb} /$ $T M 6 B, T b$, then crossing the single $P / \triangle 2-3$ males to $D r / T M 3$ females. Finally, near 200 singlecrosses of white eye male progeny $\triangle P / T M 3$ fly with $3 \mathrm{Dr} / T M 3$ virgin females were set up for excision mutation stock establishment. All the white eye recovery lines were subsequently subject to PCR screen.

\section{RESULTS AND DISCUSSION}

Seven genes (CG1463, CG17140, CG1620, CG12736, CG10214, CG31141, and 
CG13601), which were located at different chromosome loci, were used as cases for generating null alleles by recovering the inserted P-element near them (Figure 2). All the P-element insertion lines were viable except CG13601. Totally, 3, 1, 7, 1, 1, 3, and 3 alleles from 362, 751, 312, 281, 210, 370, and 277 recovery events were detected, respectively, for CG1463, CG17140, CG1620, CG12736, CG10214, CG31141, and CG13601 genes (Table 1). The deletion lines were determined by PCR. If the deletion caused by imprecise excision occurred, the PCR result would show two bands. The large one was amplified from balancer and the small one from the deletion chromosome. The small band was then purified and further confirmed by sequencing.

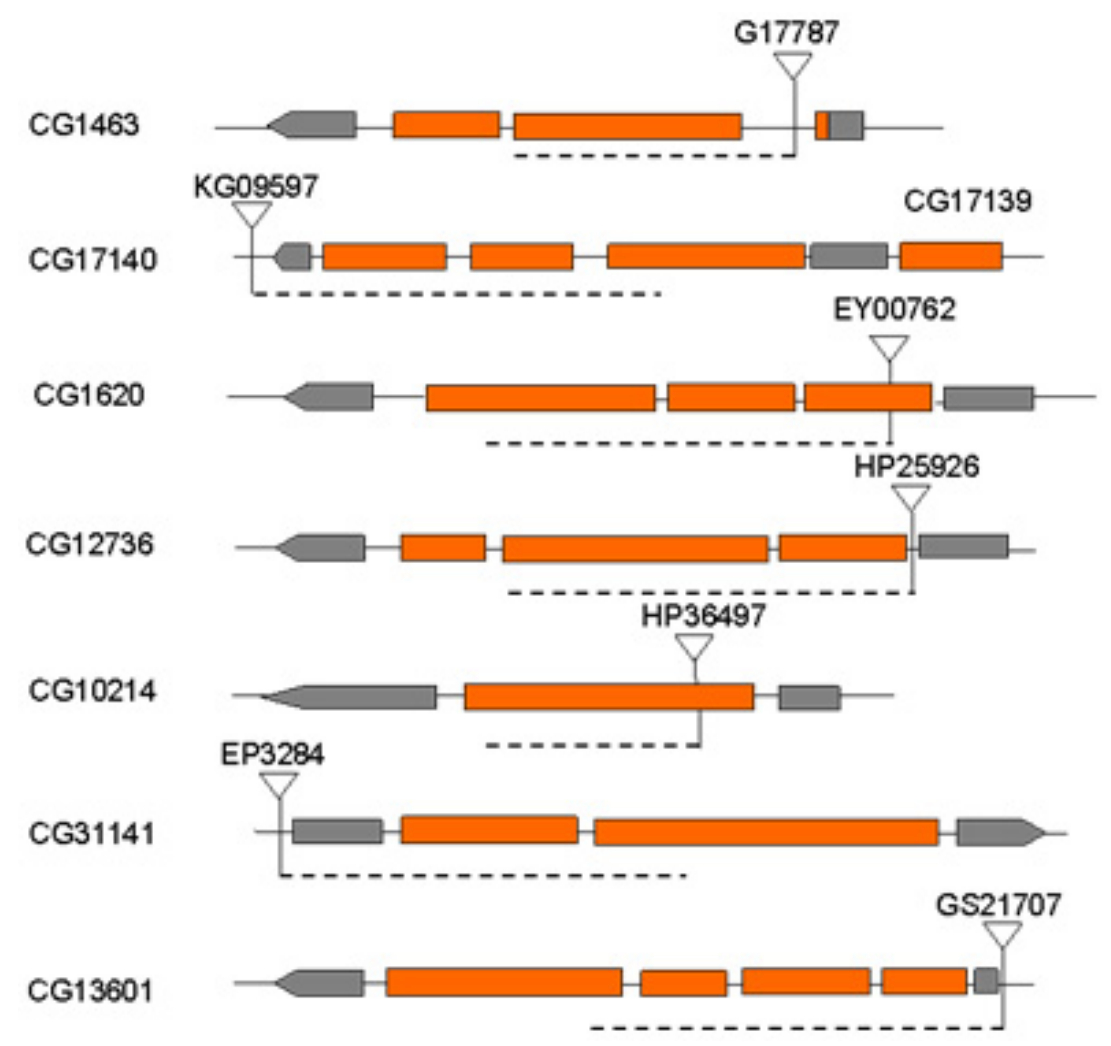

Figure 2. Structures of 7 gene loci. Transposon insertion sites (triangles), exon (red), UTR (gray). Dashed lines indicate the deleted sequences.

\begin{tabular}{|c|c|c|c|c|c|c|}
\hline Gene & Chromosome & $\begin{array}{c}\text { Recovery } \\
\text { line }\end{array}$ & $\begin{array}{l}\text { Ideal } \\
\text { allele }\end{array}$ & $\begin{array}{c}\text { Deletion } \\
\text { length (bp) }\end{array}$ & $\begin{array}{c}\text { Deletion } \\
\text { frequency (\%) }\end{array}$ & P-elements used to hopping \\
\hline CG1463 & $\mathrm{X}$ & 362 & 3 & $1231-1893$ & 0.82 & $\mathrm{w}^{*} \mathrm{P}\{\mathrm{EP}\} \mathrm{CG} 1463^{\mathrm{G} 17787}$ \\
\hline CG17140 & 2 & 751 & 1 & $386-1501$ & 0.13 & $\mathrm{y}^{1} ; \mathrm{P}\left\{\right.$ SUPor-P\}KG09597; ry ${ }^{506}$ \\
\hline CG1620 & 2 & 312 & 7 & 66-946 & 2.34 & $\mathrm{y}^{1} \mathrm{w}^{67 \mathrm{7} 23} ; \mathrm{P}\{\mathrm{EPgy} 2\} \mathrm{CG} 1620^{\mathrm{EY} 00762}$ \\
\hline CG12736 & 2 & 281 & 1 & 1601 & 0.35 & $\mathrm{w}^{1118} ; \mathrm{P}\{\mathrm{EPg}\} \mathrm{CG} 12736^{\mathrm{HP} 25926}$ \\
\hline CG10214 & 3 & 210 & 1 & 1089 & 0.47 & $\mathrm{w}^{1118} ; \mathrm{P}\{\mathrm{EPg}\} \mathrm{HP} 36497$ \\
\hline CG31141 & 3 & 370 & 3 & $1119-1300$ & 0.81 & $\mathrm{~W}^{1118} ; \mathrm{P}\{\mathrm{EP}\} \mathrm{EP} 3284$ \\
\hline CG13601 & 3 & 277 & 3 & $500-1700$ & 0.91 & $\mathrm{y}^{1} \mathrm{w}^{67 \mathrm{7c23}} ; \mathrm{P}\{\mathrm{GSV} 7\} \mathrm{GS} 21707 / \mathrm{TM} 3, \mathrm{Sb}^{1} \mathrm{Ser}^{1}$ \\
\hline
\end{tabular}


Three deletion lines were detected among 362 independent recovery events in the $\mathrm{X}$ chromosome gene CG1463. For the second chromosome genes, more than $300 \mathrm{P} / \mathrm{CyO} ;+/ 42-$ $3 \times \mathrm{Sco} / \mathrm{Cy} \mathrm{O}$ single crosses were set up, and 281 recovery lines in CG12736 were obtained. Among them, one deletion line was detected by PCR, which disrupts the major CDS. Under the same condition, however, 7 deletion lines were yielded in the CG1620 from 312 recovery lines, and one of them deleted 2/3 the CDS sequence including nearly 2.5 exons. For gene CG17140, a coding sequence of another gene CG17139 was close to its 5'-UTR. Thus, the deletion should not exceed $300 \mathrm{bp}$ to ensure the complement of CG17139 CDS. Only one such line was obtained by examining over 700 recovery flies. For the third chromosome genes CG10214, CG31141, and CG13601, 210, 370, 277 recovery $\triangle P / T M 3$ flies were yielded, respectively, and only 1, 3, 3 were identified as ideal deletion lines. Together, the results show that gene knockout by inducing P-element transposition is a feasible and effective strategy, although the frequency (ideal deletion lines/recovery lines) greatly varied, ranging from 2.34 to $0.13 \%$ for the 7 genes.

The frequency of imprecise excision varies tremendously, depending on the different kinds of P-element, the chromatin environment where the P-element is inserted, and a multitude of other factors that are not well understood. It is estimated that the proportion ranges from 35 to $75 \%$ for all recovery events (Voelker et al., 1984; O’Brochta et al., 1991; Witsell et al., 2009). Considering that in certain cases we need to create a deletion of a specific size or in a precisely defined region to disrupt a given gene through imprecise excision, the frequency should be dramatically lower. For example, the deletions can occur at either the 5'- or 3'-end of the P-element. However, we only examined one side if the P-element was inserted upstream or downstream of the gene rather within the gene sequence. Moreover, for genes that are located in the region where many genes are close together, some large deletion events that damage nearby genes should be excluded. This further lowers the knockout frequency, as in the case of CG17140. Results showed that the figure varies from $0.13-2.34 \%$. No matter how affected by these factors, on average, the frequency of flanking deletions recovered from imprecise excision screens are expected to be $1 \%$. From the experience, it is enough to pick up at least one ideal deletion line from 200 independent recovery lines in general.

Although the development of direct gene targeting by homologous recombination was a landmark breakthrough in Drosophila genetics, the procedure proved to be time-consuming and labor-intensive. This research showed that gene disruption by inducing the flanking transposon mobilization is feasible and effective. Especially, the increasing collection of various P-elements and even other transposons such as Hobo, piggyBac element as well as Minos element insertion lines make it available for more and more genes (De Freitas et al., 2008; Venken et al., 2011; Kim and Pyykko, 2011; Kim et al., 2012). Inducing imprecise excision is the first choice strategy for gene knockout. Furthermore, besides inducing single P-element transposition, we can also induce simultaneous mobilization of multiple P-elements to delete the internal sequence (Parks et al., 2004). Additionally, Witsell et al. $(2009,2010)$ recently demonstrated that it can increase the percentage of imprecise excision and overall size of flanking deletion during either $\mathrm{P}$ or Minos element transposition, when the transposition is performed in the mus309, which encodes DmBlm, a RecQ DNA helicase involved in DNA repair, mutant background. These approaches enhance the efficient desired deletion of large DNA fragment.

\section{ACKNOWLEDGMENTS}

The author thanks Dr. X.H. Lin (Institute of Zoology, Chinese Academy of Sciences) 
for fly stocks and Dr. Z.H. Yan (Columbia University, NY) for providing P-element lines. Research supported by the start-up fund from Guiyang Medical University and by the Guizhou Science and Technology Foundation (\#2012GZ81781).

\section{REFERENCES}

Cooley L, Kelley R and Spradling A (1988). Insertional mutagenesis of the Drosophila genome with single P elements. Science 239: 1121-1128.

De Freitas OM and Silva Loreto EL (2008). The hobo-related elements in the melanogaster species group. Genet. Res. 90: 243-252.

Kim A and Pyykko I (2011). Size matters: versatile use of PiggyBac transposons as a genetic manipulation tool. Mol. Cell Biochem. 354: 301-309.

Kim H, Kim K, Kim J, Kim SH, et al. (2012). Mutagenesis by imprecise excision of the piggyBac transposon in Drosophila melanogaster. Biochem. Biophys. Res. Commun. 417: 335-339.

O'Brochta DA, Gomez SP and Handler AM (1991). P element excision in Drosophila melanogaster and related drosophilids. Mol. Gen. Genet. 225: 387-394.

O'Hare K and Rubin GM (1983). Structures of P transposable elements and their sites of insertion and excision in the Drosophila melanogaster genome. Cell 34: 25-35.

Parks AL, Cook KR, Belvin M, Dompe NA, et al. (2004). Systematic generation of high-resolution deletion coverage of the Drosophila melanogaster genome. Nat. Genet. 36: 288-292.

Robertson HM, Preston CR, Phillis RW, Johnson-Schlitz DM, et al. (1988). A stable genomic source of P element transposase in Drosophila melanogaster. Genetics 118: 461-470.

Rubin GM and Spradling AC (1982). Genetic transformation of Drosophila with transposable element vectors. Science 218: 348-353.

Spradling AC, Stern D, Beaton A, Rhem EJ, et al. (1999). The Berkeley Drosophila Genome Project gene disruption project: Single P-element insertions mutating 25\% of vital Drosophila genes. Genetics 153: 135-177.

Venken KJ, Schulze KL, Haelterman NA, Pan H, et al. (2011). MiMIC: a highly versatile transposon insertion resource for engineering Drosophila melanogaster genes. Nat. Methods 8: 737-743.

Voelker RA, Greenleaf AL, Gyurkovics H, Wisely GB, et al. (1984). Frequent Imprecise Excision among Reversions of a P Element-Caused Lethal Mutation in Drosophila. Genetics 107: 279-294.

Witsell A, Kane DP, Rubin S and McVey M (2009). Removal of the bloom syndrome DNA helicase extends the utility of imprecise transposon excision for making null mutations in Drosophila. Genetics 183: 1187-1193.

Witsell A, Kane DP and McVey M (2010). Super-sized deletions: improved transposon excision screens using a mus309 mutant background. Fly 4: 137-140. 